degree of inactivation of the succinic oxidase system when different concentrations of B.A.L. were allowed to act on the enzyme for various times.

The enzyme preparation after treatment with B.A.L. could not be reactivated by catalase, cytochrome $c$, serum proteins, denatured globin, calcium phosphate gel, Straub's ' $S C$ factor'8, copper, aqueous extract of fresh heart-muscle mince (rich in myoglobin) or various fractions of the heart muscle preparation. The enzyme could, however, be protected from the action of B.A.L. by the presence, during treatment, of cytochrome $c$, which caused rapid oxidation (through the cytochrome oxidase system) of the dithiol.

Treatment with B.A.L. under conditions which caused complete inactivation of the succinic oxidase system, with about 10 per cent inhibition of succinic dehydrogenase, inhibited the activity of the system, measured anaerobically by the rate of reduction of ferricyanide, by 35 per cent.

These observations suggest the existence of a B.A.L.-labile factor, probably a hæmatin compound, which transfers electrons from cytochrome $b$ to cytochrome $c$ and is destroyed by coupled oxidation with reducing agents (cf. the action of ascorbic acid on hæmoglobin, Lemberg et al. ${ }^{9}$ ) when the heart-muscle preparation is treated with reducing agents in the presence of air.

The relationships between this factor and the cytochromes are shown in the accompanying scheme, the arrows showing direction of electron transfer. Thus destruction of the factor would not affect the oxidation of succinate through methylene blue as carrier, nor the oxidation of various reducing agents through eytochromes $c$ and $a$ and cytochrome oxidase; but would prevent completely the transfer of electrons from cytochrome $b$ to cytochrome $c$ and thence to molecular oxygen. It is postulated that potassium ferricyanide, with an oxidation-reduction potential $\left(E^{\prime}{ }_{0}\right.$ at $p \mathrm{H}$ $7 \cdot 3=+0 \cdot 41$ V.) much higher than that of succinate $\rightleftharpoons$ fumarate $\left(E^{\prime}{ }_{0}\right.$ at $p \mathrm{H}, 7 \cdot 3=-0.01 \mathrm{~V}$.) or of cytochrome $b\left(E^{\prime}{ }_{0}\right.$ at $p H 7 \cdot 3=-0.04 \mathrm{~V}$.) operates partly directly with cytochrome $b$ and partly through the B.A.L.-labile factor or the cytochromes of higher oxidation-reduction potential $\left(E^{\prime}{ }_{0}\right.$ of cytochrome $c=+0.26 \mathrm{~V}$. , cytochrome $a=+0.29$ V.). Thus the destruction of the factor causes a partial, but not complete, inhibition of the rate of oxidation by ferricyanide.

It is possible that the factor is the same as, or closely related to, the component $X$ suggested by Bach et al. ${ }^{10}$ to be necessary for the reduction of cytochrome $c$ by cytochrome $b_{2}$ in yeast.

The complete experimental data and a discussion of these findings in relation to previous work on the succinic oxidase system will appear elsewhere.

I would like to express my sincere thanks to Prof. D. Keilin for his most valuable advice throughout this investigation. I also wish to thank the British Council for a scholarship.

${ }^{2}$ Keilin, D., and Hartree, E. F., Proc. Roy. Soc., B, 129, 277 (1940). Ball, E. G., Biochem. Z., 295, 262 (1938).

Keilin, D., and Hartree, E. F., Proc. Roy. Soc., B, 127, 167 (1939).

'Hopkins, F. G., and Morgan, E. J., Biochem. J., 32, 611 (1938).

- Barron, E. S. G., Miller, Z. B., and Meyer, J., Biochem. J., 41, 78 (1947).

- Webb, E. C., and van Heyningen, R., Biochem. J., 41, 74 (1947).

'Keilin, D., and Hartree, E. F., Proc. Roy. Soc., B, 125, 171 (1938).

- Straub, F. B., Z. physiol. Chem., 272, 219 (1942).

- Lemberg, R.. Legge, J. W. and Lockwood, W. H., Biochem. J., 35, 328, 339. 353, 363 (1941).

${ }^{10}$ Bach, S. J., Dixon, M., and Zerfas, L. G., Biochem. J., 40, 229 (1946).

\section{PLANT ANALYSIS AND FERTILIZER REQUIREMENTS}

A QUICK and reliable method of determining the fertilizer requirements of crops and of discovering what nutrient deficiency is responsible for the unsatis. factory growth of some particular crop would be of immense value in advisory work. The problem can be approached from several different points of view, such as soil analysis, field trials, pot cultures, leaf injection, plant analysis and the use of visual symptoms. The relative advantages and disadvantages of these various methods of diagnosing fertilizer requirements are briefly but clearly summarized in the paper under notice*, and an extensive list of references facilitates fuller study of the individual methods.

A short historical account of the early work on diagnostic plant analysis reminds the reader that there is much to be learnt from these early papers. Developments during the past twenty-five years are discussed in more detail, and a great deal of information on the content of nutrients in plant material in relation to the incidence of deficiency or toxicity symptoms is summarized in tabular form.

Reference is also made to the use of plant analysis in the determination of current nutrient requirements by so-called 'tissue tests'. Carried out on sap or extracts of fresh material, it is suggested that the fractions estimated by these tissue tests are mainly unassimilated material which has recently entered the plant and that their concentration thus represents the current rate of nutrient intake. The rapidity with which 'tissue tests' can be performed and the fact that their results can be translated into immediate fertilizer treatment quickly enough to be effective even on annual crops, provided the problem is recognized early in the life of the crop, makes them specially attractive in advisory work. If they can be shown to be reasonably reliable and their interpretation does not require the establishment of a very elaborate series of standards, they seem likely to displace some of the older methods used in advisory work.

As the authors of this paper point out, when defining the aim of a method of deficiency diagnosis, there is an important distinction between methods to be used to improve the nutrition, during the course of its growth, of the crop analysed, and those where the plants analysed serve only as an index of the state of the soil and as a guide to the best fertilizer treatment for subsequent crops.

In a discussion of the relation of internal nutrient concentration to nutrient supply, evidence from barley grown in sand cultures, to which nutrients were added before germination, is used to show that: (1) the concentration in the tissues will depend upon the time during development at which determinations are made; and (2) the results will depend upon the part of the plant chosen for analysis. Analysis of the plant soon after germination (when the seed reserves are exhausted) will give some measure of the concentration of the nutrients in the rooting medium, especially in plants like cereals that branch very rapidly in the early stages.

The bearing of these facts on the relations to be expected with plants growing in the field are complic. ated by the difference in the mode of supply of the nutrient; for not only are the differences in the

* Chemical Composition of Plants as an Index of their Nutritional Status. (Tech. Comm. No. 17, Imperial Bureau of Horticulture and Plantation Crops.) By Dr. D.' W. Goodall and Dr. F. G. Gregory. Pp. 167. (Aberystwyth : Imp. Bur., 1947.) 98 . 
concentration of the nutrient in the field of a different order from those in the sand cultures described, but also rates at which nutrients in the soil become available are likely to be more important than actual concentrations in the soil solution, and the supply may continue at a relatively high level throughout the growing period. This is specially important in those types of plant, such as barley, in which nutrients taken up after the stage when further elaboration of meristems ceases lead to no further increase in yield but merely raise the concentration within the plant, thereby checking the normal tendency to decline to a minimum at harvest. Hence plant analyses from field crops can show considerable variations in concentration at harvest. High concentration of nutrient in the mature cereal plant may be due either to limitation of growth by some other nutrient factor leading to 'luxury consumption' or to a high supplying power of the soil maintained throughout the growing period. In the former case, the authors point out that yield will tend to be low and could be improved by the addition of the nutrient in minimum; but in the latter case yield will be high. Hence the use of analyses of mature cereal plants as indicators of the nutritional status of soils depends on the fact that differences in the nutrient-supplying power of the soil persist throughout the growing season, leading to accumulation at harvest, and not upon any causal relationship between final internal nutrient concentration and yield.

A good deal of consideration is given to the best method of selecting plants for analysis, to the influence of time of day and time of year or stage of development of the plant when samples are taken, and to the advantages of analysing the whole plant as compared with a single organ of the plant. On the subject of which organ is likely to prove the most suitable for analysis, the authors have collected all the available evidence and present it in tabular form under the various crops. In the majority of cases the leaves seem to show the greatest proportional increase in internal concentration of a nutrient with increase in its supply. Necessary precautions in the preparation of material for analysis are discussed, followed by consideration of the analysis itself, including the need to determine particular chemical fractions and the choice of extraction methods.

The interpretation of the results of diagnostic analysis is perhaps the most difficult part of the whole problem of the use of plant analysis to determ. ine fertilizer needs. Standards of reference are essential, together with data on the performance of the plants from which they are derived. To establish standard values for internal nutrient content requires data on the yield responses of plants at varying levels of nutrition and on their composition. Emphasis is placed on the dangers of basing conclusions on data for a single element without allowing for variations in the content of other nutrients. Nevertheless, it is pointed out that many investigators have specified optimal levels or limiting values above which the supply of a particular nutrient may be regarded as adequate, presumably where the content of other nutrients is not limiting. A comprehensive table of these suggested standard values for nutrient content of a wide variety of crops is included in the paper.

Suggestions by numerous workers for standard ratios of various plant nutrients are also given in tabular form; but the authors state that in their opinion there is no reason for supposing that ratios of nutrients in general are likely to be of greater use in diagnostic work than the content of the elements individually. They consider that ratios of nutrients within the plant should generally be used in conjunction with data for individual concentrations. The authors express the view that 'foliar diagnosis' work must be subject to much the same criticisms as other work involving the use of ratios.

In their final consideration of the place of plant analysis among other methods of nutritional diagnosis, the authors suggest that a combination of soil and plant analyses may give the most satisfactory results : soil analysis to determine pre-planting fertilizer treatment, and plant analysis for perennial crops and to indicate the need of annual crops for side or top dressings of fertilizer. Diagnosis by visual symptoms is an easy and rapid method where symptoms can be readily and definitely recognized, but has the disad. vantage that waiting for deficiency symptoms to appear before treatment can be given may reduce the final yield.

\section{SOCIOLOGY OF MENTAL DISEASE}

THE physical and biological sciences have at last

been accepted as indispensable adjuncts to the art of government, but the psychological and sociological lag behind. No clearer evidence of this is needed than the assumption that the men of science whose help should be asked to solve the problem of controlling atomic bombs are those who are specialists in making the machine rather than those who are specialists in the study of the minds likely to set the machine in motion.

It is encouraging, therefore, that the National Association for Mental Health should have had full and practical governmental support for its conference in the Seymour Hall during January 15-16. By sanctioning the payment of expenses, the various Ministries secured a very large attendance of delegates from local authorities. Moreover, the Parliamentary Secretary to the Minister of Health eontributed that rarest of things - an opening address which must have usefully coloured the minds of everybody throughout the ensuing meetings.

Mr. Edwards emphasized that mental illness is a social disease with sequelæ deleterious to national and international life, that its consequences affect production drives, foreign policies, the family institution, and the efficacy of education. Moreover, it is a matter which cannot be left simply to technicians, since it affects every individual, however sane potentially, since complete sanity is impossible for anyone who has to live in a community riddled with mental and nervous troubles.

Dr. G. R. Hargreaves, principal medical officer of Lever Bros. and Unilever, Ltd., and Dr. Donald Stewart, medical adviser to the Austin Motor Co., provided the text for the conference by epitomizing the Russell Fraser Report on the incidence of neurosis among factory workers*. In a sample of more than three thousand male and female workers in light and medium engineering factories, 10 per cent suffered from definite and disabling neurotic illness and a further 20 per cent from minor forms of neurosis during the course of six months. Neurotic illness accounted for a loss of three working days by every man studied and six days by every woman. It is at least as important a source of industrial loss as colds and influenza, or accidents and industrial disease.

* No. 90. Industrial Health Research Board, 1947. (H.M. Stationery Office.) $18.3 d$. 